\title{
Improvement of Treatment Outcomes for Metastatic Pancreatic Cancer: A Real-world Data Analysis
}

\author{
TAKASHI SASAKI, RYO KANATA, IKUHIRO YAMADA, \\ MASATO MATSUYAMA, MASATO OZAKA and NAOKI SASAHIRA \\ Department of Gastroenterology, Cancer Institute Hospital of Japanese \\ Foundation of Cancer Research, Tokyo, Japan
}

\begin{abstract}
Background/Aim: FOLFIRINOX (5-fluorouracil, leucovorin, irinotecan, oxaliplatin) and gemcitabine plus nabpaclitaxel therapy have recently been introduced for the treatment of metastatic pancreatic cancer. Herein, overall treatment outcomes of metastatic pancreatic cancer after introduction of FOLFIRINOX and gemcitabine plus nabpaclitaxel therapy were evaluated, in daily practice. Patients and Methods: Metastatic pancreatic cancer patients $(n=321)$ who started systemic chemotherapy between January 2011 and December 2016 were included and were divided into two groups: group A (2011-2013) and group B (2014-2016). Treatment outcomes were evaluated retrospectively. Results: Patient characteristics were similar between the two groups except for the rates of distant lymph node metastasis and peritoneal metastasis. The preferred regimens in groups $A$ and $B$ were gemcitabine monotherapy and gemcitabine plus nabpaclitaxel therapy, respectively. The response rates, median progression-free survival, and median overall survival of groups $A$ and $B$ were $7.8 \%$ and $28.4 \%(p<0.01), 3.1$ months and 5.4 months $(p<0.01)$, and 6.7 months and 10.2 months $(p<0.01)$, respectively. Conclusion: Overall treatment outcomes for metastatic pancreatic cancer were significantly improved after introduction of FOLFIRINOX and gemcitabine plus nab-paclitaxel combination therapy in daily practice.
\end{abstract}

Pancreatic cancer is the fourth leading cause of cancer-related deaths, since more than 33,000 patients are dying from this type of cancer annually in Japan (1). The prognosis of

This article is freely accessible online.

Correspondence to: Takashi Sasaki, MD, Ph.D., Department of Gastroenterology, Cancer Institute Hospital of Japanese Foundation of Cancer Research. 3-8-31, Ariake, Koto, Tokyo 135-8550, Japan. Tel: +81 335200111, Fax: +81 335200141, e-mail: sasakit-tky@umin.ac.jp

Key Words: Metastatic pancreatic cancer, chemotherapy, FOLFIRINOX, gemcitabine, nab-paclitaxel, real-world data. pancreatic cancer remains dismal, and the mortality rates closely follow the incidence rates. Surgical resection with negative margins (R0) is the only potentially curative treatment for pancreatic cancer, but only $15-20 \%$ of these patients are eligible for resection at initial diagnosis (2). More than half of patients diagnosed in the metastatic setting have a 5 -year survival rate of $2.6 \%$ (2).

Gemcitabine alone has since long been the standard chemotherapy for metastatic pancreatic cancer (3). Many randomized studies have been conducted to develop regimens that are more effective than gemcitabine alone. Combination therapy with gemcitabine and erlotinib demonstrated a significant improvement in prognosis against gemcitabine alone, but the improvement of median overall survival was only two weeks (4). Recently, combination therapy including FOLFIRINOX (5-fluorouracil, leucovorin, irinotecan, oxaliplatin) and gemcitabine plus nab-paclitaxel therapy (GEM+nab-PTX) showed superiority to gemcitabine alone (5, $6)$. Both these regimens are combination therapies of cytotoxic agents. The prognoses were prolonged by 4.3 months by FOLFIRINOX and 1.8 months by GEM+nab-PTX. Although it is difficult to compare these results directly because the study populations in each trial were different, these two regimens have become the two standard front-line treatments for metastatic pancreatic cancer.

Unfortunately, FOLFIRINOX and GEM+nab-PTX are more toxic than is gemcitabine alone $(5,6)$. To reduce the severe adverse events of FOLFIRINOX, several modified regimens have been tested and reported. In a phase II study, a modified version of FOLFIRINOX with a reduced dose of irinotecan to $150 \mathrm{mg} / \mathrm{m}^{2}$ and without the bolus of 5-fluorouracil, demonstrated a similar efficacy and reduced toxicity to FOLFIRINOX in Japanese pancreatic cancer patients (7). This modified regimen is widely used in daily practice in Japan, but this regimen remains more toxic than gemcitabine alone. Moreover, pancreatic cancer is generally a disease of the elderly, with a median age of diagnosis at 70 years, even though $40 \%$ are older than 75 years when diagnosed (8). In Japan, the median age of pancreatic cancer 
Table I. Patient characteristics.

\begin{tabular}{|c|c|c|c|}
\hline & $\begin{array}{c}\text { Group A } \\
(n=102)\end{array}$ & $\begin{array}{l}\text { Group B } \\
(n=201)\end{array}$ & $p$-Value \\
\hline Age (median, range) & $66(37-80)$ & $66(39-82)$ & 0.80 \\
\hline Gender & & & 0.63 \\
\hline Male & $58(57 \%)$ & $107(53 \%)$ & \\
\hline Female & $44(43 \%)$ & $94(47 \%)$ & \\
\hline Performance status & & & 0.37 \\
\hline 0 & $82(80 \%)$ & $147(73 \%)$ & \\
\hline 1 & $19(19 \%)$ & $51(25 \%)$ & \\
\hline 2 & $1(1 \%)$ & $3(1 \%)$ & \\
\hline Primary site & & & 0.38 \\
\hline Pancreatic head & $33(32 \%)$ & $76(38 \%)$ & \\
\hline Pancreatic body-tail & $69(68 \%)$ & $125(62 \%)$ & \\
\hline \multicolumn{4}{|l|}{ Metastatic site } \\
\hline Liver metastasis & $74(73 \%)$ & $149(74 \%)$ & 0.78 \\
\hline Lung metastasis & $15(15 \%)$ & $32(16 \%)$ & 0.87 \\
\hline Distant lymph node metastasis & $41(40 \%)$ & $51(25 \%)$ & 0.01 \\
\hline Peritoneal metastasis & $36(35 \%)$ & $39(19 \%)$ & $<0.01$ \\
\hline CEA (ng/ml) & $5.9(0.7-4,924)$ & $6.4(0-9,698.9)$ & 0.66 \\
\hline CA19-9 (U/ml) & $1,153.5(2-50,000)$ & $1,727(2-50,000)$ & 0.89 \\
\hline Biliary drainage & $21(21 \%)$ & $42(21 \%)$ & 1.00 \\
\hline
\end{tabular}

CEA, Carcinoembryonic antigen; CA19-9, carbohydrate antigen 19-9.

diagnosis is now 75 years due to the high life expectancy (1). Furthermore, there are many metastatic pancreatic cancer patients with a poor performance status (8). Consequently, these two combination therapies could be used in selected metastatic pancreatic cancer patients who maintained good performance status, while the remaining ones are still treated with gemcitabine alone. In the present study, we conducted a retrospective analysis to evaluate the improvement in treatment outcomes for metastatic pancreatic cancer patients after introduction of FOLFIRINOX and GEM+nab-PTX in their daily practice.

\section{Patients and Methods}

Patients. Patients with primary metastatic pancreatic cancer who started systemic chemotherapy between January 2011 and December 2016 at our hospital were included in this retrospective study. The patients who were recurrent after surgery were excluded from this study. In Japan, FOLFIRINOX and GEM+nab-PTX became commercially available in December 2013 and December 2014, respectively. FOLFIRINOX was first introduced at our hospital in January 2014. Therefore, the study population was divided into two groups: patients who started chemotherapy between 2011 and 2013 were as assigned to group A, and patients who started chemotherapy between 2014 and 2016 were as assigned to group B. Several clinical studies evaluating new agents as first-line chemotherapy were conducted during this period, and the patients who were included in these clinical studies were excluded from this study population. Clinical study regimens as second-line chemotherapy were allowed in this study when the patients were progressed after first-line standard chemotherapy. For FOLFIRINOX, all patients were treated with modified regimens (7) during these periods as both first-line and second-line chemotherapy.

Statistical analysis. Objective response was evaluated according to Response Evaluation Criteria in Solid Tumors (RECIST) version 1.1 (9). Progression-free survival (PFS) and overall survival (OS) were calculated using the Kaplan-Meier method, and the log-rank test for comparisons between two groups. Hazard ratio (HR) and 95\% confidence intervals were estimated by the Cox proportional hazard model. The Mann-Whitney $U$ - and Fisher's exact tests were used to compare the two groups as appropriate. The final analysis was conducted using follow-up data through the end of May 2018. Multivariate analysis was conducted to evaluate prognostic factors of metastatic pancreatic cancer by using a Cox proportional hazard model. JMP 12 statistical software (SAS Institute Inc., Cary, NC, USA) was used for all statistical analyses.

This study was conducted in compliance with the Declaration of Helsinki and according to the ethical guidelines for medical and health research involving human subjects. This study was approved by the Institutional Review Boards of our hospital (No. 2017-1140).

\section{Results}

Patient characteristics. A total of 321 consecutive patients with primary metastatic pancreatic cancer were treated with systemic chemotherapy between January 2011 and December 2016, out of which 18 were treated by a clinical study regimen. Therefore, a total of 303 patients were enrolled in this study. Pathological confirmation was achieved in $97.0 \%$ of the patients (294/303; 284 adenocarcinoma, 1 adenosquamous cell carcinoma, 8 
Table II. Treatment regimens.

\begin{tabular}{lcc}
\hline & $\begin{array}{c}\text { Group A } \\
(\mathrm{n}=102)\end{array}$ & $\begin{array}{c}\text { Group B } \\
(\mathrm{n}=201)\end{array}$ \\
\hline Gemcitabine & $83(81.3 \%)$ & $38(18.9 \%)$ \\
S-1 & $11(10.8 \%)$ & $9(4.5 \%)$ \\
Gemcitabine+S-1 & $2(2.0 \%)$ & $3(1.5 \%)$ \\
Gemcitabine+erlotinib & $6(5.9 \%)$ & 0 \\
Gemcitabine+nab-paclitaxel & 0 & $122(60.7 \%)$ \\
Modified FOLFIRINOX & 0 & $29(14.4 \%)$ \\
\hline
\end{tabular}

carcinoma, and 1 class IV by cytology). The remaining 9 patients $(3.0 \%)$, who did not have pathological evidence, were diagnosed with metastatic pancreatic cancer through the clinical course. Patient characteristics are shown in Table I. One hundred and two patients were enrolled in group A (2011-2013) and 201 patients were in group B (2014-2016). The baseline characteristics were well balanced between these two groups except for the rates of distant lymph node metastasis and peritoneal metastasis. The median age of both A and B group was 66 years. Most patients demonstrated relatively a good performance status. Treatment regimens are summarized in Table II. Gemcitabine monotherapy was the most frequently selected regimen in group A, while GEM+nab-PTX was the most frequently selected therapy used in group B. In the latter, approximately three-quarters of the patients were treated by modified FOLFIRINOX or GEM+nab-PTX. Gemcitabine and tegafur/gimeracil/oteracil potassium (S-1) combination therapy was only used in limited populations in both groups due to the results of the GEST study (10).

Treatment outcomes. The median follow-up period after treatment was 8.6 months. All final survival data were confirmed in group A. In group B, 19 patients (9.5\%) were still alive when the data were analyzed, while for 3 patients lacked final survival data $(1.5 \%)$.

Treatment outcomes are summarized in Table III. Response rates of groups A and B to treatment were $7.8 \%$ and $28.4 \%$, respectively $(p<0.01)$. Disease control rates in groups A and B were $53.9 \%$ and $65.7 \%$, respectively $(p=0.06)$. Median PFS periods in groups A and B were 3.1 months and 5.4 months, respectively $(p<0.01$; Figure 1$)$. Median OS periods in groups A and B were 6.7 months and 10.2 months, respectively $(p<0.01$; Figure 2$)$. One- and 2year survival rates in group B improved to $42.0 \%$ and $13.9 \%$ when compared to those in group A, respectively. Induction rates of second-line chemotherapy in groups $\mathrm{A}$ and $\mathrm{B}$ were $51.6 \%$ and $65.4 \%$, respectively ( $p=0.04$ ). In group A, $80.9 \%$ of patients were treated with S-1 monotherapy and $8.5 \%$ were treated with gemcitabine monotherapy. Only 1 patient

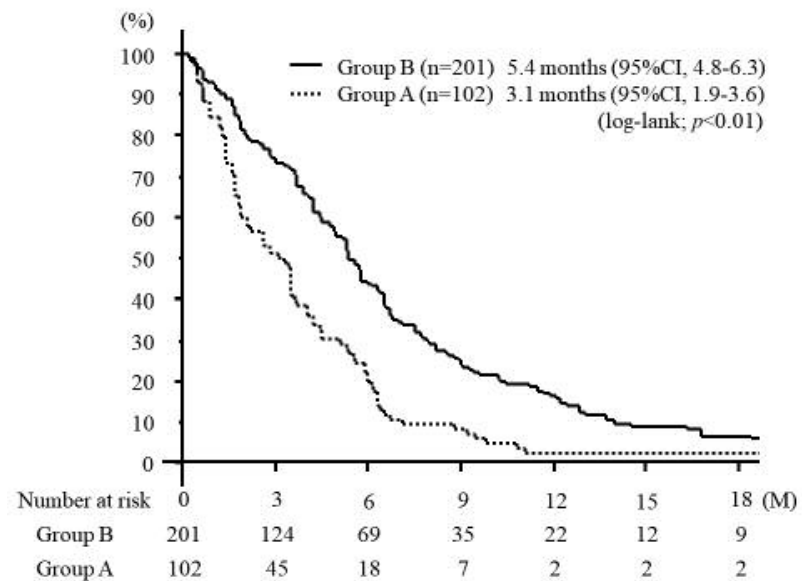

Figure 1. Progression-free survival of group A and B. 95\%CI, 95\% Confidence interval; $M$, months.

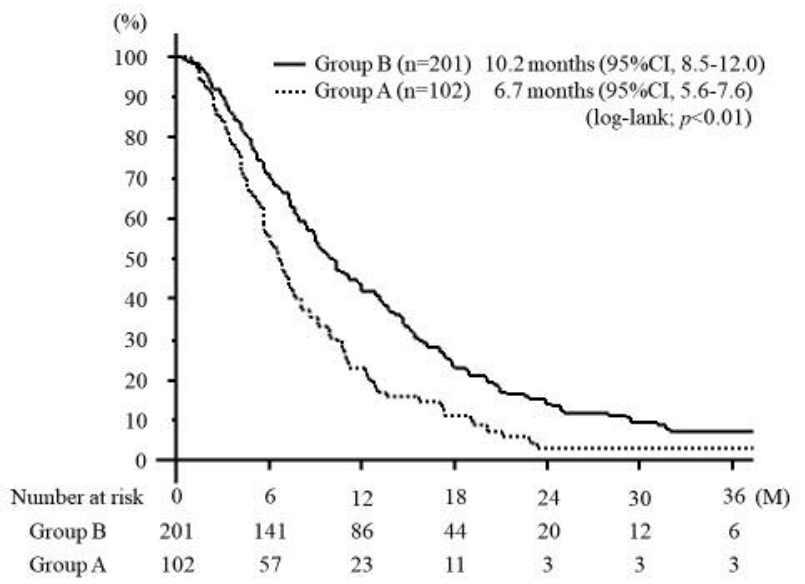

Figure 2. Overall survival of group A and B. 95\%CI, 95\% Confidence interval; $M$, months.

(2.1\%) was treated with GEM+nab-PTX, and no patient was treated with modified FOLFIRINOX in group A. In group B, $48.0 \%$ of the patients were treated with S-1 monotherapy, and modified FOLFIRINOX was selected in $26.0 \%$ of the patients. GEM+nab-PTX and gemcitabine monotherapy were chosen in $13.0 \%$ and $6.0 \%$ of patients, respectively in group B, while 6 patients $(3.0 \%)$ were offered surgical resection after first-line chemotherapy (two patients $(2 / 29 ; 6.9 \%)$ with modified FOLFIRINOX, and the remaining 4 patients $(4 / 122 ; 3.3 \%)$ were treated with GEM+nab-PTX). The median follow-up time in 6 resected patients was 36.0 months, and 5/6 patients were still alive at the time of study. No patients from group A were allowed to undergo surgical resection. 
Table III. Summary of treatment outcomes.

\begin{tabular}{lccc}
\hline & $\begin{array}{c}\text { Group A } \\
(\mathrm{n}=102)\end{array}$ & $\begin{array}{c}\text { Group B } \\
(\mathrm{n}=201)\end{array}$ & $p$-Value \\
\hline Response rate & $7.8 \%$ & $28.4 \%$ & $<0.01$ \\
Disease control rate & $53.9 \%$ & $65.7 \%$ & 0.06 \\
Progression-free survival (months) & 3.1 & 5.4 & $<0.01$ \\
6-month progression-free survival rate & $19.8 \%$ & $43.7 \%$ & 0.04 \\
1-year progression-free survival rate & $2.3 \%$ & $65.4 \%$ & $<0.01$ \\
Induction rate of second-line chemotherapy & $51.6 \%$ & 10.2 & \\
Overall survival (months) & 6.7 & $42.0 \%$ & $13.9 \%$ \\
$\quad$ 1-year survival rate & $22.6 \%$ & \\
2-years survival rate & $2.9 \%$ & & \\
\hline
\end{tabular}

Table IV. Prognostic factors of chemotherapy for metastatic pancreatic cancer.

\begin{tabular}{|c|c|c|c|c|}
\hline \multirow[t]{2}{*}{ Variables } & \multicolumn{2}{|c|}{ Univariate } & \multicolumn{2}{|c|}{ Multivariate } \\
\hline & HR $(95 \% \mathrm{CI})$ & $p$-Value & HR $(95 \% \mathrm{CI})$ & $p$-Value \\
\hline Age ( $\geq 70$ years $)$ & $1.14(0.89-1.45)$ & 0.29 & & \\
\hline Sex (male) & $1.06(0.84-1.34)$ & 0.62 & & \\
\hline Performance status $(0,1)$ & $0.19(0.08-0.63)$ & 0.01 & $0.25(0.10-0.83)$ & 0.03 \\
\hline Primary site (pancreatic head) & $1.09(0.85-1.39)$ & 0.49 & & \\
\hline Liver metastasis & $1.80(1.37-2.37)$ & $<0.01$ & $1.97(1.46-2.67)$ & $<0.01$ \\
\hline Lung metastasis & $1.02(0.73-1.39)$ & 0.91 & & \\
\hline Distant lymph node metastasis & $1.20(0.92-1.54)$ & 0.17 & & \\
\hline Peritoneal metastasis & $1.35(1.02-1.76)$ & 0.03 & $1.56(1.16-2.08)$ & $<0.01$ \\
\hline CEA & $1.04(0.17-3.30)$ & 0.96 & & \\
\hline CA19-9 & $1.62(1.17-2.21)$ & $<0.01$ & $1.47(1.05-2.03)$ & 0.03 \\
\hline Biliary drainage & $1.24(0.92-1.64)$ & 0.16 & & \\
\hline GEM+nab-PTX or mFOLFIRINOX & $0.50(0.39-0.64)$ & $<0.01$ & $0.51(0.40-0.65)$ & $<0.01$ \\
\hline
\end{tabular}

CEA, Carcinoembryonic antigen; CA19-9, carbohydrate antigen 19-9; GEM+nab-PTX, gemcitabine+nab-paclitaxel; mFOLFIRINOX, modified FOLFIRINOX; HR, hazard ratio; 95\% CI, 95\% confidence interval.

In group $\mathrm{B}$, the response rate, disease control rate, median PFS and OS of patients treated with modified FOLFIRINOX were $41.4 \%, 72.4 \%, 7.0$ months and 17.1 months, respectively. The response rate, disease control rate, median PFS and OS of patients treated with GEM+nab-PTX were $33.6 \%, 74.6 \%, 5.7$ months and 11.5 months, respectively. The response rate, disease control rate, median PFS and OS of patients treated with the other regimens in group B were $8.0 \%, 40 \%, 2.8$ months and 5.7 months, respectively.

Multivariate analysis for prognostic factors. The results of our multivariate analysis are shown in Table IV. Good performance status $(\mathrm{HR}=0.25)$ and introduction of modified FOLFIRINOX or GEM+nab-PTX as first-line chemotherapy $(\mathrm{HR}=0.51)$ emerged as better prognostic factors for metastatic pancreatic cancer. High levels of carbohydrate antigen 19-9 (CA19-9) $(\mathrm{HR}=1.47)$, the presence of liver metastasis $(\mathrm{HR}=1.97)$ and peritoneal metastasis $(\mathrm{HR}=1.56)$ emerged as poor prognostic factors.

\section{Discussion}

The overall treatment outcomes of metastatic pancreatic cancer have improved in the clinical setting due to the introduction of FOLFIRINOX and GEM+nab-PTX. More than $75 \%$ of the metastatic pancreatic cancer patients we studied were treated with modified FOLFIRINOX or GEM+nab-PTX in group B (2014-2016). In this group, tumor shrinkage was achieved more frequently compared to group A (3.6 times; $28.4 \% \mathrm{vs}$. $7.8 \%$ ) and similarly, PFS was prolonged to 5.4 months, achieving a 2.3-month extension. Median OS was prolonged 
from 6.7 months to 10.2 months and the 1-year survival rate was $42.0 \%$. Approximately $14 \%$ of metastatic pancreatic cancer patients in group B survived for more than 2 years.

Because of the better efficacies of modified FOLFIRINOX and GEM+nab-PTX, 6 patients (3.0\%) in group $\mathrm{B}$ had surgical resection. In the era before FOLFIRINOX and GEM+nab-PTX, it was extremely rare that patients with metastatic pancreatic cancer would undergo surgical resection (11). The median follow-up time of 6 resected patients was 36.0 months, and 5/6 patients were still alive. Although a long-term follow up is necessary to validate the efficacy of conversion surgery for metastatic pancreatic cancer, the introduction of efficacious chemotherapeutic regimens is undoubtedly a great opportunity that allows conversion surgery in these patients. The major criteria of conversion surgery in our hospital are: i) disappearance of metastatic lesions; ii) resectable primary lesion; iii) normalization of tumor markers; and iv) duration of chemotherapy for at least eight months. The last criterion was chosen based on data from a study conducted by the Japanese Society of HepatoBiliary-Pancreatic Surgery (11). We believe that the criteria for conversion surgery need to be stricter for metastatic pancreatic cancer than for locally advanced pancreatic cancer, thus chemotherapy was sometimes extended for 1 year. Therefore, only a stringent group of selected patients underwent surgical resection even after the introduction of FOLFIRINOX and GEM+nab-PTX.

Improvements in median PFS and OS between groups A and B were 2.3 months and 3.5 months, respectively. The difference between the PFS and OS was only 1.2 months, suggesting that the impact of second-line chemotherapy remained limited, even in group B (2014-2016). The most widely used second-line chemotherapy in group B was S-1 monotherapy $(48.0 \%)$, while treatment with modified FOLFIRINOX (26.0\%) was second best. Even in the modified regimen of FOLFIRINOX, only a few patients were able to benefit from it. Therefore, a less toxic yet effective second-line chemotherapy is needed. Nanoliposomal irinotecan with fluorouracil and folinic acid might be a good option for patients having second-line chemotherapy (12). Quite importantly, an effective third-line chemotherapy should be available to prolong the OS of metastatic pancreatic cancer patients.

There was a trend towards improvement in the disease control rate of group B, but it was not statistically significant compared to group A $(p=0.06)$. The disease control rate in group B was only $65.7 \%$. Many patients in this group were diagnosed at extremely advanced stages and could not continue the treatment long enough even if first-line chemotherapy had already been introduced. Early diagnosis is essential to resolve this problem, and educational activities for citizens regarding pancreatic cancer are still needed. Moreover, the median age of diagnosing pancreatic cancer in Japan is now 75 years because of high life expectancy (1). Approximately $33 \%$ of the pancreatic cancer patients were older than 80 years of age when diagnosed in Japan (1). More than $20 \%$ of the patients in group B chose monotherapy even though treatment with GEM+nab-PTX is relatively tolerable. Therefore, some new less toxic regimens are required to resolve this problem. It is also very important to establish standard treatment for erderly patients with metastatic pancreatic cancer (13-14).

Limitations of this study include that the data were collected from a single cancer center and were retrospectively analyzed. There were several treatment regimens involved in this study, and it is difficult to compare the adverse events. Therefore, it is not clear whether prolonging the survival time was concomitant with maintaining a good quality of life. There is a possibility that quality of life deteriorated due to the adverse events of FOLFIRINOX or GEM+nab-PTX, as it has been reported previously (14). Peripheral neurotoxicity is now a substantial problem in the management of pancreatic cancer patients who are treated with these chemotherapies $(5,6,15)$. It is important to establish the management of this side-effect and to develop new effective chemotherapies with different profiles of adverse events (16-20).

In conclusion, overall treatment outcomes of chemotherapy for metastatic pancreatic cancer were significantly improved after introduction of FOLFIRINOX and GEM+nab-PTX in the daily practice. However, about $25 \%$ of the metastatic pancreatic cancer patients could not be treated with these two effective regimens as first-line chemotherapy. Furthermore, only less than $40 \%$ of the patients were treated with these two regimens as second-line chemotherapy. More effective secondline and third-line chemotherapy regimens with less toxicity are required to prolong the overall survival of metastatic pancreatic cancer patients.

\section{References}

1 Cancer Registry and Statistics: Cancer Information Service, National Cancer Center, Japan. 2018. https://ganjoho.jp/ reg_stat/statistics/dl/index.html. Accessed October 2018.

2 Ilic M and Ilic I: Epidemiology of pancreatic cancer. World J Gastroenterol 22: 9694-9705, 2016.

3 Burris HA 3rd, Moore MJ, Andersen J, Green MR, Rothenberg ML, Modiano MR, Cripps MC, Portenoy RK, Storniolo AM, Tarassoff P, Nelson R, Dorr FA, Stephens CD and Von Hoff DD: Improvements in survival and clinical benefit with gemcitabine as first-line therapy for patients with advanced pancreas cancer: a randomized trial. J Clin Oncol 15: 2403-2413, 1997.

4 Moore MJ, Goldstein D, Hamm J, Figer A, Hecht JR, Gallinger S, Au HJ, Murawa P, Walde D, Wolff RA, Campos D, Lim R, Ding K, Clark G, Voskoglou-Nomikos T, Ptasynski M, Parulekar W; National Cancer Institute of Canada Clinical Trials Group: Erlotinib plus gemcitabine compared with gemcitabine alone in patients with advanced pancreatic cancer: a phase III trial of the National Cancer Institute of Canada Clinical Trials Group. J Clin Oncol 25: 1960-1966, 2007. 
5 Conroy T, Desseigne F, Ychou M, Bouché O, Guimbaud R, Bécouarn Y, Adenis A, Raoul JL, Gourgou-Bourgade S, de la Fouchardière $\mathrm{C}$, Bennouna $\mathrm{J}$, Bachet $\mathrm{JB}$, Khemissa-Akouz $\mathrm{F}$, Péré-Vergé D, Delbaldo C, Assenat E, Chauffert B, Michel P, Montoto-Grillot C, Ducreux M; Groupe Tumeurs Digestives of Unicancer; PRODIGE Intergroup: FOLFIRINOX versus gemcitabine for metastatic pancreatic cancer. N Engl J Med 364: 1817-1825, 2011.

6 Von Hoff DD, Ervin T, Arena FP, Chiorean EG, Infante J, Moore M, Seay T, Tjulandin SA, Ma WW, Saleh MN, Harris M, Reni M, Dowden S, Laheru D, Bahary N, Ramanathan RK, Tabernero J, Hidalgo M, Goldstein D, Van Cutsem E, Wei X, Iglesias J and Renschler MF: Increased survival in pancreatic cancer with nabpaclitaxel plus gemcitabine. N Engl J Med 369: 1691-1703, 2013.

7 Ozaka M, Ishii H, Sato T, Ueno M, Ikeda M, Uesugi K, Sata N, Miyashita K, Mizuno N, Tsuji K, Okusaka T and Furuse J: A phase II study of modified FOLFIRINOX for chemotherapynaïve patients with metastatic pancreatic cancer. Cancer Chemother Pharmacol 81: 1017-1023, 2018.

8 Sohal DP, Mangu PB, Khorana AA, Shah MA, Philip PA, O'Reilly EM, Uronis HE, Ramanathan RK, Crane $\mathrm{CH}$, Engebretson A, Ruggiero JT, Copur MS, Lau M, Urba S and Laheru D: Metastatic pancreatic cancer: American Society of Clinical Oncology Clinical Practice Guideline. J Clin Oncol 34: 2784-2796, 2016.

9 Eisenhauer EA, Therasse P, Bogaerts J, Schwartz LH, Sargent D, Ford R, Dancey J, Arbuck S, Gwyther S, Mooney M, Rubinstein L, Shankar L, Dodd L, Kaplan R, Lacombe D and Verweij J: New response evaluation criteria in solid tumours: revised RECIST guideline (version 1.1). Eur J Cancer 45: 228247, 2009.

10 Ueno H, Ioka T, Ikeda M, Ohkawa S, Yanagimoto H, Boku N, Fukutomi A, Sugimori K, Baba H, Yamao K, Shimamura T, Sho M, Kitano M, Cheng AL, Mizumoto K, Chen JS, Furuse J, Funakoshi A, Hatori T, Yamaguchi T, Egawa S, Sato A, Ohashi Y, Okusaka T and Tanaka M: Randomized phase III study of gemcitabine plus S-1, S-1 alone, or gemcitabine alone in patients with locally advanced and metastatic pancreatic cancer in Japan and Taiwan: GEST study. J Clin Oncol 31: 1640-1648, 2013.

11 Satoi S, Yamaue H, Kato K, Takahashi S, Hirono S, Takeda S, Eguchi H, Sho M, Wada K, Shinchi H, Kwon AH, Hirano S, Kinoshita T, Nakao A, Nagano H, Nakajima Y, Sano K, Miyazaki M and Takada T: Role of adjuvant surgery for patient with initially unresectable pancreatic cancer with a long-term favorable response to non-surgical anti-cancer treatments: results of a project study for pancreatic surgery by the Japanese Society of Hepato-Biliary-Pancreatic Surgery. J Hepatobiliary Pancreat Sci 20: 590-600, 2013.

12 Wang-Gillam A, Li CP, Bodoky G, Dean A, Shan YS, Jameson G, Macarulla T, Lee KH, Cunningham D, Blanc JF, Hubner RA, Chiu CF, Schwartsmann G, Siveke JT, Braiteh F, Moyo V, Belanger B, Dhindsa N, Bayever E, Von Hoff DD, Chen LT; NAPOLI-1 Study Group: Nanoliposomal irinotecan with fluorouracil and folinic acid in metastatic pancreatic cancer after previous gemcitabine-based therapy (NAPOLI-1): a global, randomized, open-label, phase 3 trial. Lancet 387: 545-557, 2016.
13 Ishii H, Yamashita N, Ueno M, Ohkawa S, Saito AM and Sekimoto M: A randomized controlled trial of gemcitabine hydrochloride plus S-1 combination therapy versus gemcitabine hydrochloride therapy alone in pancreatic cancer patients aged $\geq 75$ years: a study protocol for an open-label randomized feasibility study. BMJ Open Gastroenterol 5: e000187, 2018.

14 Betge J, Chi-Kern J, Schulte N, Belle S, Gutting T, Burgermeister E, Jesenofsky R, Maenz M, Wedding U and Ebert MP, N: A multicenter phase 4 geriatric assessment directed trial to evaluate gemcitabine $+/-$ nab-paclitaxel in elderly pancreatic cancer patients (GrantPax). BMC Cancer 18: 747, 2018.

15 Goldstein D, Von Hoff DD, Moore M, Greeno E, Tortora G, Ramanathan RK, Macarulla T, Liu H, Pilot R, Ferrara S and Lu B: Development of peripheral neuropathy and its association with survival during treatment with nab-paclitaxel plus gemcitabine for patients with metastatic adenocarcinoma of the pancreas: A subset analysis from a randomized phase III trial (MPACT). Eur J Cancer 52: 85-91, 2016.

16 Ma J, Kavelaars A, Dougherty PM and Heijnen CJ: Beyond symptomatic relief for chemotherapy-induced peripheral neuropathy: Targeting the source. Cancer 124: 2289-2298, 2018.

17 Smith EM, Pang H, Cirrincione C, Fleishman S, Paskett ED, Ahles T, Bressler LR, Fadul CE, Knox C, Le-Lindqwister N, Gilman PB, Shapiro CL; Alliance for Clinical Trials in Oncology: Effect of duloxetine on pain, function, and quality of life among patients with chemotherapy-induced painful peripheral neuropathy: a randomized clinical trial. JAMA 309: 1359-1367, 2013.

18 Scheithauer W, Ramanathan RK, Moore M, Macarulla T, Goldstein D, Hammel P, Kunzmann V, Liu H, McGovern D, Romano A and Von Hoff DD: Does modification and efficacy of nab-paclitaxel plus gemcitabine $v s$. gemcitabine for patients with metastatic pancreatic cancer: phase III MPACT trial. J Gastrointest Oncol 7: 469-478, 2016.

19 Relias V, Maloney A, Smith MH and Saif MW: Does "OPTINAB" strategy ("stop-and-go") work in treatment of advanced pancreatic cancer (APC) with nab-paclitaxelgemcitabine? Cancer Chemother Pharmacol 80: 371-375, 2017.

20 Tong H, Fan Z, Liu B and Lu T: The benefits of modified FOLFIRINOX for advanced pancreatic cancer and its induced adverse events: a systematic review and meta-analysis. Sci Rep 8: 8666, 2018.
Received October 8, 2018

Revised November 11, 2018 Accepted November 15, 2018 